\title{
Notes on the Riemann zeta-function, II
}

\author{
by
}

K. Ramachandra (Bangalore) and A. Sankaranarayanan (Mumbai)

Dedicated with deepest regards to Professor Alan Baker, F.R.S., on his sixtieth birthday

1. Introduction. Let $N$ and $D$ be two finite sets of complex numbers defined by

$$
N \equiv\left\{\alpha_{1}, \ldots, \alpha_{k}\right\}, \quad D \equiv\left\{\beta_{1}, \ldots, \beta_{l}\right\}
$$

where $k \geq 0, l \geq 1, k \leq l$ and $\Sigma^{*} \equiv \sum_{j=1}^{k, *} \operatorname{Re}\left(\beta_{j}-\alpha_{j}\right)<1$ where the asterisk denotes the sum over only positive terms. We stress here that neither the $\alpha$ 's nor the $\beta$ 's need be distinct. Let $P(s)$ be any finite Dirichlet series. Then, defining the empty product to be 1 , we have the following theorem.

Theorem. Let $F(s)$ defined by

$$
F(s) \equiv\left(P(s) \prod_{\alpha \in N} \zeta(s+\alpha)\right)\left(\prod_{\beta \in D} \zeta(s+\beta)\right)^{-1} \equiv F_{1}(s)\left(F_{2}(s)\right)^{-1}
$$

(with an obvious notation) be a non-terminating Dirichlet series for $\operatorname{Re}(s) \geq$ $1000+\sum|\alpha|+\sum|\beta|$. Then $F(s)$ has infinitely many poles in $|\operatorname{Im}(s)| \geq c$ for every fixed $c>0$. Furthermore the result is still true if we replace $F(s)$ by any finite sum of functions of the type $F(s)$ (for various $P(s), N$ and $D$ ).

REMARK 1. In our theorem some (or all) of $\zeta(s+\alpha)$ can be replaced by their derivatives of bounded order.

Remark 2. Here some (or all) of $\zeta(s+\beta)$ can be replaced by the corresponding ordinary $L$-functions. At the same time some (or all) of $\zeta(s+\alpha)$ can be replaced by the derivatives of bounded order of $L(s+\alpha)$ for ordinary $L$-functions or those of $\sum_{n=1}^{\infty}(a n+b)^{-s-\alpha}$ where $a, b$ are positive integers.

REMARK 3. Here some (or all) of $\zeta(s+\beta)$ can be replaced by $L^{*}(s+\beta)$ where $L^{*}$ are the $L$-functions of quadratic fields. At the same time some (or

1991 Mathematics Subject Classification: 11M06, 11M41. 
all) of $\zeta(s+\alpha)$ can be replaced by $L^{*}(s+\alpha)$, or $\zeta^{*}(s+\alpha)$ (where $\zeta^{*}$ are zeta-functions of ray classes of quadratic fields) or derivatives of these of any bounded order. The result is true with an obvious modification of the condition $\Sigma^{*}<1$.

REMARK 4. This paper arose out of an attempt to generalize a result of E. C. Titchmarsh (see Theorem 14.27 on p. 372 of [T]). We can also state results corresponding to the divergence of $\sum\left|\varrho \zeta^{\prime}(\varrho)\right|^{-1}$. Titchmarsh makes some assumptions like the Riemann hypothesis and for the divergence problem he needs the simplicity of the zeros of $\zeta(s)$. Our results do not depend on any unproved hypothesis.

REMARK 5. In many of our results above we can solve the following problem (see $\S 6$ ). Given any $T_{1}>0$, we can find an explicit $T_{2}>T_{1}$ such that there is at least one pole $\varrho$ of $F(s)$ satisfying

$$
T_{1}<\operatorname{Im}(\varrho)<T_{2} .
$$

Combining this with a method of R. Balasubramanian and K. Ramachandra (see [BR1]) we can solve the following problem effectively. Let

$$
F(s)=\sum_{n=1}^{\infty} a_{n} n^{-s}, \quad \operatorname{Re}(s)>1000+\sum|\alpha|+\sum|\beta|,
$$

for any of the functions above. Suppose that $a_{n}$ are all real and that $F(s)$ does not have a pole with real $s>0$. Put $\operatorname{Re}(\varrho)=\Theta$ for the pole $\varrho$ above. Then $\sum_{n \leq x} a_{n}=\Omega_{ \pm}\left(x^{\Theta}\right)$ provided $\Theta>0$. Note that if the poles of $F(s)$ are situated symmetrically with respect to the half line then we can take $\Theta \geq 1 / 2$ and so definitely $\Theta=1 / 2$. In particular we can prove things like (here $\Lambda(n)$ is the von Mangoldt function)

$$
\sum_{\substack{n \leq x \\ n \equiv 1(\bmod 5)}} \Lambda(n)-\sum_{\substack{n \leq x \\ n \equiv-1(\bmod 5)}} \Lambda(n)=\Omega_{ \pm}\left(x^{1 / 2}\right),
$$

and so

$$
\sum_{\substack{p \leq x \\ p \equiv 1(\bmod 5)}} \log p-\sum_{\substack{p \leq x \\ p \equiv-1(\bmod 5)}} \log p=\Omega_{ \pm}\left(x^{1 / 2}\right)
$$

which are effective. No doubt such results are already in the famous works of S. Knapowski and P. Turán who founded the subject "comparative Prime Number Theory". But our theorem is more in the spirit of generalization. One may note that this theorem enables us to prove things like (here $\mu(n)$ is the Möbius function)

$$
\sum_{\substack{n \leq x \\ n \equiv 1(\bmod 5)}} \mu(n)=\Omega_{ \pm}\left(x^{1 / 2}\right)
$$


REMARK 6 . We are unable to put our theorem in such a way as to include the case $F(s)=\left(\zeta^{\prime}(s)\right)^{2}(\zeta(s))^{-1}$. In this case the poles of $F(s)$ are precisely the simple zeros of $\zeta(s)$. A simple ingenious proof of infinitude of the simple zeros of $\zeta(s)$ in $\operatorname{Im}(s)>0$ is due to J. B. Conrey, A. Ghosh and S. M. Gonek [CGG]; see also Section 5.4 of [R1].

REMARK 7. We conjecture that the results mentioned in the Theorem (and also in the remarks above) are valid without the condition $\Sigma^{*}<1$. For our results in this direction see Section 6.

Corollary. The functions $\zeta(s+\sqrt{-1})(\zeta(s))^{-1}, \zeta^{\prime}(s+\sqrt{-1})(\zeta(s))^{-1}$ and $(\zeta(s))^{-1} \pm(L(s))^{-1}$, where $L(s)=\sum_{n=1}^{\infty}(-1)^{n-1}(2 n-1)^{-s}$ and its analytic continuation, have infinitely many poles in $\operatorname{Im}(s) \geq 10$, whatever be the combination \pm .

2. Notation. We adopt the standard notation of the subject. Usually we use $C_{1}, C_{2}, \ldots$ to denote large positive constants. The triple line $\equiv$ denotes a definition. Vinogradov's symbols $\ll$ and $\gg$ denote "less than a constant times", and "greater than a constant times" respectively. The symbol $O(\ldots)$ means $\ll \ldots$ The symbol $\asymp$ means both $\ll$ and $\gg$ together. The symbol $\Omega$ means $\gg$ infinitely often. If $f$ and $g$ are two real-valued functions then $f=$ $\Omega_{+}(g)$ means $f$ exceeds a positive constant times $g$ infinitely often, and $f=$ $\Omega_{-}(g)$ means that $-f=\Omega_{+}(g)$. The symbol $\Omega_{ \pm}$means both $\Omega_{+}$and $\Omega_{-}$.

3. Sketch of the proof of the Theorem. As regards the second assertion of the Theorem it suffices to prove it with $F(s)$ replaced by

$$
\left(\sum\left\{P(s) \prod_{\alpha \in N} \zeta(s+\alpha)\right\}\right)\left(\prod_{\beta \in D} \zeta(s+\beta)\right)^{-1}
$$

where $\sum$ denotes a finite sum of terms similar to the ones in the curly brackets (for various $P$ 's and $N$ 's). This can be seen by simplifying the sum of fractions in question to a common denominator. The treatment of (1) is not very much different from that of the first assertion of the Theorem. For simplicity we assume $P(s)=1$ and consider only the first assertion of the Theorem. Regarding $F_{1}(s)$ we need only the result

$$
\frac{1}{T} \int_{T}^{2 T}\left|\zeta\left(\frac{1}{2}+i t\right)\right|^{2} d t=O\left((\log T)^{C_{1}}\right) \quad(T \geq 10) .
$$

From (2) it follows that

$$
\int_{1 / 2-\Delta}^{2}\left(\frac{1}{T} \int_{T}^{2 T}|\zeta(\sigma+i t)|^{2} d t\right) d \sigma=O\left((\log T)^{C_{1}+10}\right)
$$

where $\Delta=(\log T)^{-1}$ (these results are well known). 
Such results hold for $\left[T, T+T^{1 / 3}\right]$ in place of $[T, 2 T]$. We need (2) only when $F_{1}(s) \neq 1$. The relations (2) and (3) are definitely true for functions mentioned in Remarks 1 to 3. Also whenever such functions have an Euler product, then $N(\sigma, T)$ denoting the number of zeros (of such functions) with $\operatorname{Re}(s) \geq \sigma$ and $|\operatorname{Im}(s)| \leq T$, we have, by standard methods,

$$
N(\sigma, T) \leq T^{3(1-\sigma) /(4-2 \sigma)}(\log T)^{C_{2}} \quad(T \geq 10,1 / 2 \leq \sigma \leq 1) .
$$

The inequality (4) is useful to deal with $F_{2}(s)$. Apart from some usual techniques (to deal with $F_{1}(s)$ ) the main difficulty is to deal with $F_{2}(s)$ and here we follow the pattern set out in [RS].

From now on we write $s=\sigma+i t, i=\sqrt{-1}$. The main idea is to start with

$$
\sum_{n \leq x} a_{n}=\frac{1}{2 \pi i} \int_{\sigma_{1}-i T}^{\sigma_{1}+i T} F(s) \frac{x^{s}}{s} d s+O\left(T^{-1}\right)
$$

(where $\sigma_{1}=1000+\sum|\alpha|+\sum|\beta|, x$ is a large positive constant $\geq 100$ and we adopt the convention that whenever $x$ is an integer the last term in the sum on the LHS is to be halved), where the $a_{n}$ are defined by

$$
F(s)=\sum_{n=1}^{\infty} a_{n} n^{-s} \quad\left(\text { for } \sigma \geq \sigma_{1}\right) .
$$

Put

$$
\begin{aligned}
J & =\frac{1}{2 \pi i} \int_{\sigma_{1}-i T}^{\sigma_{1}+i T} F(s) \frac{x^{s}}{s} d s \\
& =\frac{1}{2 \pi i}\left\{J_{1}+J_{2}+J_{3}+J_{4}+J_{5}\right\}+S
\end{aligned}
$$

where

$$
\begin{aligned}
J_{1} & \equiv \int_{\sigma_{1}-i T}^{-\sigma_{1}-i T} \ldots, \\
J_{2} & \equiv \int_{-\sigma_{1}-i T}^{-N-i T} \ldots, \\
J_{3} & \equiv \int_{-N-i T}^{-N+i T} \ldots, \\
J_{4} & \equiv \int_{-N+i T}^{-\sigma_{1}+i T} \ldots,
\end{aligned}
$$




$$
J_{5} \equiv \int_{-\sigma_{1}+i T}^{\sigma_{1}+i T} \ldots
$$

and

$$
S=\text { sum of the residues of } F(s) \frac{x^{s}}{s} \text { in the relevant rectangle. }
$$

Here $N$ should not be confused with that introduced first in the introduction. $N\left(\geq \sigma_{1}\right)$ and $T(\geq 10)$ are such that the paths of integration in the equations (9) to (13) are bounded away from the singularities of $F(s)$ to the extent possible. First we choose $N=N_{\nu}(\nu=1,2, \ldots)$ such that as $N \rightarrow \infty$,

$$
J_{3} \rightarrow 0
$$

for every fixed $T$ provided $x$ exceeds a large positive constant. Having proved this we next prove that as $N \rightarrow \infty$ we have

$$
\left.\left|J_{2}\right|+\left|J_{4}\right|=O\left(T^{-1+\Sigma^{*}+\varepsilon}\right) \quad \text { (for every } \varepsilon>0\right) .
$$

These follow nearly as in the proof of Theorem 14.27 on p. 372 of [T]. But the greatest difficulty is to prove

$$
\left|J_{1}\right|+\left|J_{5}\right|=O\left(T^{-1+\Sigma^{*}+\varepsilon}\right) \quad(\text { for every } \varepsilon>0)
$$

for a suitable sequence $T=T_{\nu}^{(0)} \rightarrow \infty$. We show that this is possible for every large $X$ and suitable $T_{\nu}^{(0)}$ with $X \leq T_{\nu}^{(0)} \leq 2 X$. For this purpose we select certain $t$-intervals $I \subset[X, 2 X]$ for which (on $\left(-\sigma_{1} \leq \sigma \leq \sigma_{1}\right) \times I$ ) we know good upper bounds for $\zeta(s)$ and its derivatives and further

$$
\min _{t \in I} \max _{\sigma \geq 1 / 2}\left(\max _{\beta \in D}|\zeta(\sigma+i t+i \operatorname{Im}(\beta))|^{-1}\right) \leq\left(\exp (\log \log X)^{2}\right)^{C_{3}} .
$$

From these it follows that the sum on the LHS of (5) is the same as $S$, which is a continuous function of $x$ (for large $x$ ) if there are no poles of $F(s)$ in $|t| \geq C$ (for some $C>0$ ). This proves our Theorem provided (15)-(17) are established.

The remainder of the proof will be arranged as follows. In Section 4 we prove (15) and (16). The proof of (17) is a long story; we devote Section 5 to it. In (16) and (17) it may be noted that the quantity $T^{\varepsilon}$ can be replaced by a suitable positive constant power of $\exp \left((\log \log T)^{2}\right)$. In Section 6 we make some concluding remarks about effectiveness of our results.

4. Proof of (15) and (16). In treating $J_{2}, J_{3}$ and $J_{4}$, the main results that we use are

$$
\zeta(1-s)=2(2 \pi)^{-s} \cos (s \pi / 2) \Gamma(s) \zeta(s)
$$

$$
\Gamma(s)=\sqrt{2 \pi} s^{s-1 / 2} e^{-s}\left(1+O\left(|s|^{-1}\right)\right)
$$


the latter being valid in $\left(\left|\tan ^{-1}(t / \sigma)\right| \leq \pi-\pi / 1000, \sigma \geq 2\right)$. We first treat $J_{3}$ (defined by (11)). Clearly

$$
J_{3}=J_{3}^{\prime} \equiv \int_{N+1-i T}^{N+1+i T} \frac{x^{1-s} F(1-s)}{1-s} d s .
$$

Now

$$
F(1-s)=\left(P(1-s) \prod_{\alpha} \zeta(1-s+\alpha)\right)\left(\prod_{\beta} \zeta(1-s+\beta)\right)^{-1} .
$$

(Note that the set $N$ in $\alpha \in N$ is omitted to avoid confusion with $N$ in $N+1 \pm i T$.) Trivially

$$
|P(1-s)| \leq P_{0}^{N}
$$

where $P_{0} \geq 2$ is some constant. We take care of the other factors by the following two lemmas.

Lemma 1. For $s=N+1+i t,|t| \leq T$, and $w$ a complex variable subject to $|w| \leq|\log s|^{-1}$, we have the inequality

$$
\begin{aligned}
|\zeta(1-s-w+\alpha)| \ll & (2 \pi)^{-N} e^{(\pi / 2)|t|-N}|N+i t|^{N-\operatorname{Re}(\alpha)+1 / 2} \\
& \times \exp \left(-|t-\operatorname{Im}(\alpha)| \tan ^{-1} \frac{|t-\operatorname{Im}(\alpha)|}{N+1}\right) .
\end{aligned}
$$

Hence by Cauchy's theorem,

$$
\zeta^{(\nu)}(1-s+\alpha)=O\left((\mathrm{RHS})|\log s|^{\nu}\right)
$$

for the derivatives of $\zeta(1-s+\alpha)$ of order $\nu \geq 0$.

Proof. We have by (19) and (20),

$$
|\zeta(1-s-w+\alpha)| \ll(2 \pi)^{-N} e^{(\pi / 2)|t|}|\Gamma(s+w-\alpha)| .
$$

Now $|\Gamma(s)| \asymp|\exp (-s+(s-1 / 2) \log s)|$ and so

$$
|\Gamma(s+w-\alpha)| \asymp|\Gamma(s-\alpha)|
$$

and

$$
\begin{aligned}
|\Gamma(s-\alpha)| \asymp & e^{-N}\left|\exp \left((s-\alpha-1 / 2)\left(\log |s-\alpha|+i \tan ^{-1} \frac{t-\operatorname{Im}(\alpha)}{N+1}\right)\right)\right| \\
\asymp & e^{-N} \mid \exp ((N+1-\operatorname{Re}(\alpha)+1 / 2) \log |N+i t| \\
& \left.-(t-\operatorname{Im}(\alpha)) \tan ^{-1} \frac{t-\operatorname{Im}(\alpha)}{N+1}\right) \mid .
\end{aligned}
$$

This together with

$$
\frac{1}{\nu !} \zeta^{(\nu)}(1-s+\alpha)=\frac{1}{2 \pi i} \int_{|w|=|\log s|^{-1}} \zeta(1-s-w+\alpha) w^{-\nu-1} d w
$$

proves Lemma 1. 
Lemma 2. For $s=N+1+i t,|t| \leq T$, we have

$$
\begin{aligned}
|\zeta(1-s+\beta)|^{-1} \ll & (2 \pi)^{N} e^{-(\pi / 2)|t|+N}|N+i t|^{-N+\operatorname{Re}(\beta)-1 / 2} \\
& \times \exp \left(|t-\operatorname{Im}(\beta)| \tan ^{-1} \frac{|t-\operatorname{Im}(\beta)|}{N+1}\right)
\end{aligned}
$$

provided $\left|\cos \left(\frac{\pi}{2}(s-\beta)\right)\right|^{-1} \ll e^{-(\pi / 2)|t|}$. The last inequality can be secured for all $\beta$ uniformly if we alter $N$ by a small quantity.

Proof. This follows from Lemma 1.

We are now in a position to prove the following lemma.

Lemma 3. For fixed $T \geq 10$ and all $N \geq T^{2}$ we have the estimate

$$
J_{3}=J_{3}^{\prime}=O\left(T x^{-N} P_{1}^{N}\right)
$$

where $P_{1} \geq 2$ is some constant. Thus if $x \geq 2 P_{1}$, the quantity $J_{3} \rightarrow 0$ as $N \rightarrow \infty$ provided $T$ is fixed.

REMARK. In the $O$-estimate the factor $(N T)^{\varepsilon}(\varepsilon>0$ arbitrary) becomes necessary if we want to allow for $\zeta^{(\nu)}(s+\alpha)$.

Proof (of Lemma 3). Using Lemmas 1 and 2 we have the following estimates for all $N \geq T^{2}$ :

$$
\begin{aligned}
|F(1-s)| \leq & P_{0}^{N}(2 \pi)^{-N(k-l)} e^{(k-l)((\pi / 2)|t|-N)}|N+i t|^{(k-l)(N+1 / 2)} \\
& \times|N+i t|^{\sum \operatorname{Re}(\beta)-\sum \operatorname{Re}(\alpha)}<P_{1}^{N}
\end{aligned}
$$

where (since $k \leq l) P_{1} \geq 2$ is some constant. Thus $J_{3}=J_{3}^{\prime}=O\left(T x^{-N} P_{1}^{N}\right)$ and this proves the lemma.

We now prove the following lemma.

LEMMA 4. We have

$$
\left|J_{2}\right|+\left|J_{4}\right|=O\left(T^{-1+\Sigma^{*}}\right)
$$

where the $O$-constant is independent of $T$ and $N$.

REMARK. The factor $T^{\varepsilon}(\varepsilon>0$ arbitrary) becomes necessary if we want to allow for $\zeta^{(\nu)}(s+\alpha)$.

Pro of (of Lemma 4). It suffices to prove the estimate for $J_{2}$ (the proof for $J_{4}$ being similar). Clearly

$$
J_{2}=\int_{-\sigma_{1}-i T}^{-N-i T} F(s) \frac{x^{s}}{s} d s=-J_{2}^{\prime},
$$

where

$$
J_{2}^{\prime} \equiv \int_{1+\sigma_{1}+i T}^{1+N+i T} \frac{F(1-s) x^{1-s}}{1-s} d s
$$


For $s=\sigma+i T, 1+\sigma_{1} \leq \sigma \leq N+1$ we have

$$
F(1-s)=\left(P(1-s) \prod_{\alpha} \zeta(1-s+\alpha)\right)\left(\prod_{\beta} \zeta(1-s+\beta)\right)^{-1} .
$$

Now for all $T \geq 2 \sigma_{1}$ and all $\sigma$ we have

and

$$
\begin{aligned}
\exp (-(T- & \left.\operatorname{Im}(\alpha)) \tan ^{-1} \frac{T-\operatorname{Im}(\alpha)}{\sigma+1}\right) \\
& \asymp \exp \left(-T \tan ^{-1} \frac{T-\operatorname{Im}(\alpha)}{\sigma+1}\right) \asymp \exp \left(-T \tan ^{-1} \frac{T}{\sigma+1}\right)
\end{aligned}
$$

$$
\begin{aligned}
\exp \left((T-\operatorname{Im}(\beta)) \tan ^{-1} \frac{T-\operatorname{Im}(\beta)}{\sigma+1}\right) & \asymp \exp \left(T \tan ^{-1} \frac{T-\operatorname{Im}(\beta)}{\sigma+1}\right) \\
& \asymp \exp \left(T \tan ^{-1} \frac{T}{\sigma+1}\right)
\end{aligned}
$$

since

$$
\begin{aligned}
\left|\tan ^{-1} \frac{T-\operatorname{Im}(\alpha)}{\sigma+1}-\tan ^{-1} \frac{T}{\sigma+1}\right| & =\left|\int_{T}^{T-\operatorname{Im}(\alpha)} \frac{\sigma+1}{u^{2}+(\sigma+1)^{2}} d u\right| \\
& \ll\left|\int_{T}^{T-\operatorname{Im}(\alpha)} \frac{|u(\sigma+1)|}{u^{2}+(\sigma+1)^{2}} \frac{d u}{u}\right| \ll \frac{1}{T} .
\end{aligned}
$$

Thus for some constant $P_{2} \geq 2$,

$$
\begin{aligned}
|F(1-s)| \ll & P_{2}^{\sigma}(2 \pi)^{-\sigma(k-l)} e^{((\pi / 2) T-\sigma)(k-l)} \\
& \times \prod_{\alpha}|\sigma+i T|^{\sigma-\operatorname{Re}(\alpha)+1 / 2} \prod_{\beta}|\sigma+i T|^{-\sigma+\operatorname{Re}(\beta)-1 / 2} \\
& \quad \times \exp \left((l-k) T \tan ^{-1} \frac{T}{\sigma+1}\right) \\
\ll & P_{2}^{\sigma} e^{-\sigma(k-l)}|\sigma+i T|^{(k-l)(\sigma+1 / 2)+\sum \operatorname{Re}(\beta)-\sum \operatorname{Re}(\alpha)} \\
& <P_{3}^{\sigma}|\sigma+i T|^{(k-l)(\sigma+1 / 2)+\sum \operatorname{Re}(\beta)-\sum \operatorname{Re}(\alpha)}
\end{aligned}
$$

where $P_{3} \geq 2$ is some constant. If $l \geq k+1$ then the exponent of $|\sigma+i T|$ in the last expression is negative and so the last expression does not exceed $P_{3}^{\sigma}$. If $l=k$ then $\sum \operatorname{Re}(\beta)-\sum \operatorname{Re}(\alpha) \leq \Sigma^{*}$ and in all cases

$$
\left|J_{2}\right| \ll \int_{1+\sigma_{1}}^{1+N}\left(P_{3} x^{-1}\right)^{\sigma}|\sigma+i T|^{\Sigma^{*}-1} d \sigma \leq T^{\Sigma^{*}-1} \int_{1+\sigma_{1}}^{\infty}\left(P_{3} x^{-1}\right)^{\sigma} d \sigma
$$

since $\Sigma^{*}<1$ by assumption. Thus if $x \geq P_{3}^{2}$ we have

$$
\left|J_{2}\right| \ll T^{\Sigma^{*}-1} \text {. }
$$

This completes the proof of Lemma 4. 
5. Proof of (17). We begin with a few lemmas of which many are of interest in themselves.

Lemma 5. Put $\Delta=(\log T)^{-1}$. Then

$$
\int_{1 / 2-\Delta}^{2}\left(\int_{T}^{2 T}|\zeta(\sigma+i t)|^{2} d t\right) d \sigma \ll T \log \log T
$$

Pro of. The assertion follows from

$$
\left|\zeta(\sigma+i t)-\sum_{n \leq 10 T} n^{-\sigma-i t}\right| \ll T^{-\sigma} \quad(0 \leq \sigma \leq 2, T \geq 10, T \leq t \leq 2 T),
$$

where the implied constant is absolute. This result is well known (see (4.11.1) on p. 77 of Titchmarsh's book [T]). See also [BR2] for an alternative approach to this result.

Lemma 6. Let $10 \Delta \leq M \leq T^{1 / 2}$ and let $I$ run over all disjoint subintervals (of length $M$ ) of $[T, 2 T]$ excluding at most one subinterval. Let $m(I)$ denote the maximum of $|\zeta(\sigma+i t)|$ in $[1 / 2,2] \times I$. Then

$$
\sum_{I}(m(I))^{2} \ll \Delta^{-2} T \log \log T .
$$

Pr o of. From Cauchy's theorem we can prove (see [R1], Theorem 1.7.1) that

$$
(m(I))^{2} \leq \frac{1}{\pi \Delta^{2}} \iint_{D_{0}}|\zeta(\sigma+i t)|^{2} d a
$$

where the integration is over the disc $D_{0}$ of radius $\Delta$ and centre being the point of the rectangle $[1 / 2,2] \times I$ at which $m(I)$ is attained. Here $d a$ is the element of area. Summing up over all intervals $I$ we obtain

$$
\sum_{I}(m(I))^{2} \leq \frac{2}{\pi \Delta^{2}} \int_{1 / 2-\Delta}^{2}\left(\int_{T-\Delta}^{2 T+\Delta}|\zeta(\sigma+i t)|^{2} d t\right) d \sigma=O\left(\Delta^{-2} T \log \log T\right) .
$$

This proves the lemma.

LEMMA 7. We have, for some I, the estimate

$$
m(I)=O\left(\Delta^{-1} M^{1 / 2}(\log \log T)^{1 / 2}\right) .
$$

In particular, choosing $M=10 \Delta$, we obtain

$$
\min _{T \leq t \leq 2 T} \max _{1 / 2 \leq \sigma \leq 2}|\zeta(\sigma+i t)|=O\left((\log T)^{1 / 2}(\log \log T)^{1 / 2}\right) .
$$

Choosing $M=(\log T)^{C_{4}}$, we have

$$
m(I) \ll(\log T)^{(1 / 2) C_{4}+1}(\log \log T)^{1 / 2},
$$

for at least one interval I of length $M$. 
For our purposes, we record

Corollary to Lemma 6. Let $M=(\log T)^{D_{1}}$ where $D_{1}$ is any positive constant $\geq 3$. Then the number $N_{1}$ of intervals $I$ (of length $M$ ) with $m(I)>$ $(\log T)^{E}$, where $E>0$ is any constant, satisfies

$$
N_{1}=O\left(T(\log T)^{3-2 E}\right)
$$

and the total number of such intervals is $\asymp T(\log T)^{-D_{1}}$.

Proof. This follows from Lemma 6 .

LEMMA 8. We have, with the notation of (4),

$$
N(\sigma, T) \ll T^{(4(1-\sigma)) /(3-2 \sigma)}(\log T)^{100}
$$

where $1 / 2 \leq \sigma \leq 1, T \geq 10$ and the implied constant is absolute.

Proof. This is (4) with $C_{2}=100$ and this result is well known (see Titchmarsh's book [T] and also Ramachandra's book [R2]).

Lemma 9. Put $\sigma_{2}=1 / 2+G(\log \log T)(\log T)^{-1}$, where $G$ is a large positive constant. Let $D_{1}$ be as in Corollary to Lemma 6 . Then

$$
N\left(\sigma_{2}, 2 T\right)-N\left(\sigma_{2}, T\right) \ll T(\log T)^{100-G}
$$

and so if $N_{2}$ is the number of subintervals $I$ such that the rectangle $\left[\sigma_{2}, 1\right] \times I$ contains a zero of $\zeta(s)$, then

$$
N_{2}=O\left(T(\log T)^{100-G}\right) .
$$

Proof. This follows from

$$
\begin{aligned}
\frac{4(1-\sigma)}{3-2 \sigma} & =1-\left(1-\frac{4-4 \sigma}{3-2 \sigma}\right)=1-\frac{2 \sigma-1}{3-2 \sigma} \\
& \leq 1-\frac{2 \sigma-1}{2}=1-\left(\sigma-\frac{1}{2}\right) .
\end{aligned}
$$

LEMma 10. With the exception of $O\left(T(\log T)^{3-2 E}+T(\log T)^{100-G}\right)$ subintervals $I(\subset[T, 2 T])$ of length $(\log T)^{D_{1}}$ we have the following results:

$$
\zeta(s) \neq 0 \quad \text { in }\left[\sigma_{2}, 1\right] \times I
$$

and also

$$
|\zeta(s)| \leq(\log T)^{E} \quad \text { in }[1 / 2,2] \times I .
$$

Trivially the total number of such subintervals is $\leq T(\log T)^{-D_{1}}+1$.

From (41) and the functional equation it follows that

$$
|\zeta(s)|=O\left(T^{1 / 2-\sigma}(\log T)^{E}\right) \quad \text { in }\left[-\sigma_{1}, 1 / 2\right] \times I .
$$


Hence by Cauchy's theorem, namely ( $k$ not to be confused with that in $N$ in the introduction)

$$
\frac{1}{k !} \zeta^{(k)}\left(s_{0}\right)=\frac{1}{2 \pi i} \int_{\left|s-s_{0}\right|=\left(\log \left|s_{0}\right|\right)^{-1}} \zeta(s)\left(s-s_{0}\right)^{-k-1} d s
$$

valid in $\operatorname{Im}\left(s_{0}\right) \geq 10$, we have

$$
\left|\zeta^{(k)}(s)\right|=O\left(T^{1 / 2-\sigma}(\log T)^{E+k+3}\right)
$$

and

$$
\left|\zeta^{(k)}(s)\right|=O\left((\log T)^{E+k+3}\right)
$$

valid respectively in $\left[-\sigma_{1}, 1 / 2\right] \times I$ and $[1 / 2,2] \times I$, the upper and lower portions of length 1 being deleted from these rectangles so as to accommodate the application of (43). We denote by $I_{1}$ the corresponding intervals $I$. We further delete from $I_{1}$ intervals of length $\log T$ on both sides so as to accommodate $\zeta^{(k)}(s \pm i \operatorname{Im}(\alpha))(\alpha \in N)$ (for both signs) and $\zeta^{(k)}(s \pm i \operatorname{Im}(\beta))$ $(\beta \in D)$ (for both signs) in (44) and (45) and also to have

$$
H(s) \equiv \prod_{\beta \in D} \zeta(s+i \operatorname{Im}(\beta)) \zeta(s-i \operatorname{Im}(\beta)) \neq 0
$$

in $\left[\sigma_{2}, 1\right] \times I_{2}$ where $I_{2}$ are the new intervals. Further we delete intervals of length $(\log T)^{2}$ on both sides of $I_{2}$ and call them $I_{3}$. Let $k \geq 1$ be an integer (not to be confused with $k$ in $N$ of the introduction). Writing

$$
(H(s))^{k} \equiv \sum_{n=1}^{\infty} b_{n} n^{-s}
$$

we approximate LHS by $\sum_{n=1}^{\infty} b_{n} e^{-n / X} n^{-s}$ for $s$ in $\left[\sigma_{2}, 1\right] \times I_{3}$ and proceed as in Lemmas 1 to 8 of our previous paper [RS] with the same title as the present one to obtain the following lemma.

Lemma 11. We have

$$
\min _{I_{3}} \min _{t \in I_{3}} \max _{\sigma \geq 1 / 2}|H(s)|^{-1} \leq\left(\exp (\log \log T)^{2}\right)^{C_{5}} .
$$

LEMMA 12. Let

$$
K(s)=\sum_{\beta \in D}|\zeta(s+i \operatorname{Im}(\beta))|^{-1}+\sum_{\beta \in D}|\zeta(s-i \operatorname{Im}(\beta))|^{-1} .
$$

Then there exists an interval $I_{3}$ for which

$$
\min _{t \in I_{3}} \max _{\sigma \geq 1 / 2}|K(s)| \leq\left(\exp (\log \log T)^{2}\right)^{C_{6}} .
$$

Proof. Put

$$
H(s) K(s)=Q(s) .
$$


Let $t \in I_{3}$, the interval for which $\min _{I_{3}} \ldots$ in (46) is attained. Hence the expression $Q(s)$ in (49) can be estimated to be $(\log T)^{C_{7}}$ by our remarks following Lemma 10. This proves Lemma 12.

Lemma 13. In $J_{1}$ and $J_{5}$, we have

$$
|F(s)| \ll T^{\Sigma^{*}+\varepsilon} .
$$

REMARK. In fact $T^{\varepsilon}$ can be replaced by a positive constant power of $\left(\exp (\log \log T)^{2}\right)$.

Pr o of (of Lemma 13). Combining Lemmas 10 and 12, we have

$$
|F(s)| \ll T^{\lambda+\varepsilon}
$$

where

$$
\lambda=\sum_{j=1}^{k}\left(\max \left(1 / 2-\sigma-\operatorname{Re}\left(\alpha_{j}\right), 0\right)-\max \left(1 / 2-\sigma-\operatorname{Re}\left(\beta_{j}\right), 0\right)\right) .
$$

If now $-\operatorname{Re}\left(\alpha_{j}\right) \leq-\operatorname{Re}\left(\beta_{j}\right)$, then for such $j$ the terms of the sums are not positive. On the other hand, if $-\operatorname{Re}\left(\alpha_{j}\right)>-\operatorname{Re}\left(\beta_{j}\right)$, i.e. $\operatorname{Re}\left(\alpha_{j}\right)<\operatorname{Re}\left(\beta_{j}\right)$, then for such $j$ the terms are

$$
\begin{aligned}
\operatorname{Re}\left(\beta_{j}-\alpha_{j}\right)+\max \left(1 / 2-\sigma-\operatorname{Re}\left(\beta_{j}\right), \operatorname{Re}\left(\alpha_{j}-\beta_{j}\right)\right) & \\
& -\max \left(1 / 2-\sigma-\operatorname{Re}\left(\beta_{j}\right), 0\right) \\
\leq \operatorname{Re}\left(\beta_{j}-\alpha_{j}\right)+\max \left(1 / 2-\sigma-\operatorname{Re}\left(\beta_{j}\right), 0\right) & -\max \left(1 / 2-\sigma-\operatorname{Re}\left(\beta_{j}\right), 0\right)
\end{aligned}
$$

and the lemma follows.

This completes the proof of the Theorem.

REMARK 1. This has relevance to Remark 3 following our Theorem. If we assume the estimate

$$
T^{-2 / 3} \int_{T}^{T+T^{2 / 3}}\left|\zeta^{*}\left(\frac{1}{2}+i t\right)\right| d t \ll(\log T)^{D_{2}}
$$

(where $D_{2}$ is some positive constant and $\zeta^{*}(s)$ is the zeta-function of a ray class in a quadratic field; this result is due to M. Jutila $[\mathrm{J}]$ ), then in place of $X \leq T_{\nu}^{(0)} \leq 2 X$ we can have $X \leq T_{\nu}^{(0)} \leq X+X^{2 / 3}(\log X)^{-100}$. If however we do not assume the above estimate (which is very deep), then we can have $X \leq T_{\nu}^{(0)} \leq X+X(\log X)^{-100}$.

REMARK 2. This has relevance to Remarks 1 and 2 following our Theorem. Here we can have $T_{\nu}^{(0)}$ with $X \leq T_{\nu}^{(0)} \leq X+X^{1 / 3}(\log X)^{-100}$.

REMARK 3. In the above remarks the constant 100 is unimportant. We can have any positive constant in place of 100 . 
REMARK 4. If we assume the Riemann hypothesis we can have $T_{\nu}^{(0)}$ with $X \leq T_{\nu}^{(0)} \leq X+X^{\delta}$ (for every fixed $\delta>0$ ).

REMARK 5. In connection with our Theorem, we here recall the "explicit formula"

$$
\left|S-\sum_{n \leq x} a_{n}\right|=O\left(\left(T_{\nu}^{(0)}\right)^{-1+\Sigma^{*}+\varepsilon}\right)
$$

which satisfies the above requirements according to various conditions. Note that $S$ depends upon $T_{\nu}^{(0)}$. Here in the $O$-estimate $\left(T_{\nu}^{(0)}\right)^{\varepsilon}$ can be replaced by a positive constant power of $\left(\exp (\log \log T)^{2}\right)$.

6. Concluding remarks. Probably the following hypothesis is true of all functions $F(s)$ in our Theorem and in the remarks following it whenever $F(s)\left(\operatorname{Re}(s) \geq \sigma_{1}\right)$ is a non-terminating Dirichlet series (even on ignoring the condition $\left.\Sigma^{*}<1\right)$.

Hypothesis. There exist effective constants $C_{8}, C_{9}$ and $C_{10}$ such that for all $y \geq C_{8}$,

$$
\max \left|a_{n}\right| \geq n^{-C_{9}}
$$

where the maximum is taken over all $n$ with $y \leq n \leq y^{C_{10}}$.

Using this hypothesis we solve the following problem:

Problem. Given any $T_{1} \geq 100$, determine effectively a $T_{2}>T_{1}$ such that there exists a pole @ of $F(s)$ satisfying

$$
T_{1} \leq \operatorname{Im}(\varrho) \leq T_{2} .
$$

Solution. Let $T_{3}$ and $T_{4}$ be two of the numbers $T_{\nu}^{(0)}(\nu=1,2, \ldots)$ such that

$$
X \leq T_{3} \leq 2 X \quad \text { and } \quad 4 X \leq T_{4} \leq 8 X .
$$

Denote by $R$ the rectangle $\left[-T_{3}, T_{3}\right] \times\left[T_{3}, T_{4}\right]$. Suppose there are no poles of $F(s)$ in $R$. Then we derive a contradiction as follows: Now $F(s)$ is analytic in $R$. Let $s \in B$, the boundary of $R$. By our results of Sections 4 and 5 we have

$$
|F(s)|<X^{\Phi} \quad \text { on } B
$$

and hence by the maximum-modulus principle we have

$$
|F(s)|<X^{\Phi} \quad \text { for all } s \in R
$$

Here $X$ exceeds a large constant and $\Phi$ is some positive constant. Hence by the third main theorem on p. 51 of [R1], we have 


$$
\left(\frac{9}{10} T_{4}-\frac{11}{10} T_{3}\right)^{-1} \int_{(11 / 10) T_{3}}^{(9 / 10) T_{4}}|F(\sigma+i t)|^{2} d t \gg n^{-2 \sigma}\left|a_{n}\right|^{2}
$$

where $|\sigma| \leq(1 / 2) T_{3}$ and $n$ is any integer such that $X^{1-\varepsilon} \geq n \geq X^{A^{-1}}$, $A>1$ is any large positive constant. By our hypothesis there exists an $n$ in this range for which $\left|a_{n}\right| \geq n^{-C_{9}}$. Thus (selecting a suitable $n$ given by the hypothesis)

$$
\max _{(11 / 10) T_{3} \leq t \leq(9 / 10) T_{4}}|F(\sigma+i t)| \gg n^{-\sigma}\left|a_{n}\right| \geq n^{-C_{9}-\sigma}>\left(X^{A^{-1}}\right)^{-C_{9}-\sigma} .
$$

Now by choosing $\sigma$ to be a large negative constant we see that

$$
\left(X^{A^{-1}}\right)^{-C_{9}-\sigma}>X^{\Phi+1} \text {. }
$$

This contradiction proves what we want, namely (52).

Similarly there exists another pole with imaginary part between $-T_{2}$ and $-T_{1}$.

REMARK. Of course we can give (avoiding the deep result of [R1] mentioned above due to R. Balasubramanian and K. Ramachandra) a selfcontained proof of these results using the Montgomery-Vaughan theorem (see $[\mathrm{MV}])$. But it is convenient to use the Balasubramanian-Ramachandra result.

Acknowledgements. During the investigations of this paper the first author was a senior scientist of the NIAS. The second author was able to complete this work during his visit to NIAS and he is grateful to NIAS for its kind hospitality. The authors are grateful to Professor A. Schinzel for getting the paper typed in tex. They are also grateful to the referee for correcting a serious oversight and for pointing out that some work in this direction has been done by E. Grosswald [G] and later also by J. Kaczorowski and A. Perelli $[\mathrm{KP}]$. In these two references the authors have used upper bounds for things like

$$
\max \left|\frac{d^{n}}{d s^{n}} \log \zeta(s)\right| \text { and } \max \left|\frac{d^{n}}{d s^{n}} \log L(s, \chi)\right|
$$

on certain horizontal lines. (Both the papers deal with applications to comparative prime number theory.) The essential point of difference in our work are the sharp upper bounds [RS] for things like

$$
\max |\zeta(s)|^{-1} \text { and } \max |L(s, \chi)|^{-1}
$$

on certain horizontal lines. Also we have established in the present paper simultaneity results for things like

$$
\max |\zeta(s+\alpha)|^{-1} \text { and } \max |L(s+\alpha, \chi)|^{-1}
$$

(for a finite set of complex constants $\alpha$ ) on certain horizontal lines. 


\section{References}

[BR1] R. Balasubramanian and K. Ramachandra, Some problems of analytic number theory II, Studia Sci. Math. Hungar. 14 (1997), 193-202.

[BR2] - - , On an analytic continuation of $\zeta(s)$, Indian J. Pure Appl. Math. 18 (1987), 790-793.

[CGG] J. B. Conrey, A. Ghosh and S. M. Gonek, Simple zeros of zeta-functions, in: Colloq. de Théorie Analytique des Nombres, Université de Paris Sud, 1985, 77-83.

[G] E. Grosswald, Sur une propriété des racines complexes des fonctions $L(s, \chi)$, C. R. Acad. Sci. Paris 260 (1965), 4299-4302.

[J] M. Jutila, The fourth power moment of the Riemann zeta-function over a short interval, in: Number Theory, Vol. I (Budapest, 1987), Colloq. Math. Soc. János Bolyai 51, North-Holland, Amsterdam, 1990, 221-244.

[KP] J. Kaczorowski and A. Perelli, Functional independence of the singularities of a class of Dirichlet series, Amer. J. Math. 120 (1998), 289-303.

[MV] H. L. Montgomery and R. C. V aughan, Hilbert's inequality, J. London Math. Soc. (2) 8 (1974), 73-82.

[R1] K. Ramachandra, On the Mean-Value and Omega-Theorems for the Riemann Zeta-Function, Tata Inst. Fund. Res. Lectures on Math. and Phys. 85, Springer, 1995.

[R2] -, Riemann Zeta-Function, Ramanujan Institute, University of Madras, Chennai, 1979 (a pamphlet, 16 pp.).

[RS] K. Ramachandra and A. Sankaranarayanan, Notes on the Riemann zetafunction, I, J. Indian Math. Soc. 57 (1991), 67-77.

[T] E. C. Titchmarsh, The Theory of the Riemann Zeta-Function, 2nd ed., revised and edited by D. R. Heath-Brown, Clarendon Press, Oxford, 1986.

N.I.A.S., I.I.Sc. Campus

Bangalore 560 012, India

and

T.I.F.R. Centre, I.I.Sc. Campus

P.O. Box 1234

Bangalore-560 012, India

E-mail: kram@math.tifrbng.res.in
School of Mathematics Tata Institute of Fundamental Research

Homi Bhabha Road Mumbai 400 005. India E-mail: sank@math.tifr.res.in 